\title{
Effectiveness of a multicomponent intervention on the screen time of Brazilian adolescents: non-randomized controlled study
}

\author{
Jéssika Aparecida Jesus Vieira ${ }^{1}$, Luiz Rodrigo Augustemak de Lima ${ }^{1}$, Diego Augusto Santos Silva ${ }^{1}$, \\ Edio Luiz Petroski ${ }^{1}$ \\ ${ }^{1}$ Universidade Federal de Santa Catarina, UFSC, Research Center in Kinanthropometry \\ and Human Performance, Florianópolis, SC, Brazil
}

\begin{abstract}
Aim: The aim of this study was to verify the effectiveness of a multicomponent, school-based intervention in reducing screentime in adolescents according to sex. Methods: This is a non-randomized controlled intervention study focusing on cardiorespiratory fitness, body image, nutrition and physical activity. Screentime (television, computer/ video game) was the secondary outcome of the intervention and was addressed using educational strategies (folders, posters, educational sessions and group discussions). Screen time was measured using a structured questionnaire validated in Brazilian adolescents and the excess time spent on-screen was defined as two hours or more daily. The effect of the intervention was analyzed by the McNemar test and logistic regression. Results: Intervention effectively reduced the proportion of adolescents exposed to more than two hours a day of computer/videogame in males from $71.7 \%$ to $57.5 \%(\mathrm{p}=0.002)$ and excessive time of television in females from $81.5 \%$ to $72.6 \%(\mathrm{p}=0.024)$, from the beginning to the end of the study in the group receiving intervention. However, no effect of intervention was found in the comparison between intervention and control groups for computer/video game time $(\mathrm{OR}=0.822 ; \mathrm{p}=0.504)$ and television time $(\mathrm{OR}=0.667 ; \mathrm{p}=0.252)$. Conclusion: The intervention was not effective in reducing screen time in the comparison between intervention and control groups, but it reduced the screen time of adolescents in the intervention group.
\end{abstract}

Keywords: adolescents, interventions study, sedentary lifestyle.

\section{Introduction}

Excessive screen time ( $\geq 2$ hours), regardless of level of physical activity, is associated with an increased risk of developing cardiometabolic diseases, psychological problems and all causes of death ${ }^{1-3}$. American children and adolescents spend between 40 and $60 \%$ of their time on sedentary behavior ${ }^{4}$. A study conducted in 43 countries in Europe and North America found that $56.0 \%$ of 11 -year-old adolescents spent more than two hours a day watching television; among adolescents aged ${ }^{13-15}$ years, percentages were $65 \%$ and $63 \%$, respectively. In Brazil, a population-based study conducted with adolescents in the 9th grade of high school found that $78.6 \%$ watched television for two or more hours daily ${ }^{6}$. In this context, the literature indicates the need for interventions with educational actions and strategies that include school, family and health professionals to increase physical activity levels and reduce sedentary behavior?

Interventions have been developed in several countries and have reached different results regarding the effectiveness of reducing screen time. Some studies with adolescents of both sexes did not reduce the television, computer and videogame time $^{8-12}$. Successful interventions have in common the fact that they have been developed in developed and high-income countries. Interventions developed with female adolescents using educational strategies have reduced the time spent on the computer ${ }^{13}$. Programs developed with educational sessions, videos and games have reduced computer time on weekdays ${ }^{14}$, and programs developed to prevent obesity using educational lessons and practical physical activity sessions decreased the time spent in front of the television ${ }^{15}$. Interventions applied to female adolescents and developed with educational sessions to increase physical activity and reduce sedentary behavior have reduced the use of television ${ }^{16}$. These effective schoolbased interventions to reduce screen time have been applied in different countries and it was observed that among them, the use of educational strategies is a consensus.

School interventions in Brazil have presented divergent results on the effectiveness in reducing screen time in adolescents. Based on educational strategies such as seminars and group discussions, recess practical activities and extra Physical Education (PE) sessions, an intervention with girls reduced computer and video game time by 15 minutes on weekend days ${ }^{17}$, but did not reduce the television time. Another intervention reduced the average hours in front of the television, computer or video game from 4.1 to 3.3 hours per day ${ }^{18}$. Intervention to increase physical activity, performed with high school adolescents using environmental modifications and physical activity sessions did not reduce screen-time ${ }^{19}$. Although the development of some interventions to reduce screen time in Brazil have shown positive results, they have been restricted to subgroups, applied only to female adolescents, older children or adolescents, which demonstrates the need for interventions that contemplate the heterogeneity in the school environment. 
It was observed that successful national and international interventions in reducing screen time have limited actions to subgroups such as women and children. Thus, the available information makes it difficult to implement and develop interventions in the school environment, which presents a wide age range of variability in both sexes. In addition, the multicomponent character of the approach of actions and subjects is important, considering the various contexts in which adolescents are inserted, making it necessary to direct actions to the family, school and friends, intervening in basically all social circles of the adolescent. In view of the aforementioned gaps, more multicomponent interventions with effective strategies for reducing screen time in adolescents 20 are needed, supported by behavior change theories that consider the particularities of a developing country, such as Brazil. Thus, the aim of the present study was to verify the effectiveness of a multicomponent intervention in reducing the screen time of Brazilian adolescents according to sex (male/female). Our hypothesis was that adolescents of both sexes belonging to the intervention group would reduce exposure to screen time when compared to the control group.

\section{Methods}

\section{Study Design}

This is a non-randomized, controlled trial developed in two schools of the municipal teaching network of Florianópolis, in southern Brazil. The "Mexa-se - De mãos dadas pela saúde" intervention had as primary outcomes health-related physical fitness and body image of adolescents (Figure 1).

\section{Ethical aspects}

The macroproject is registered with the National Institute of Health (http://www.clinicaltrials.gov/: NCT02719704) and also in the Brazilian Registry of Clinical Trials (REBEC). The study protocol was approved by the Ethics Committee in Research with Human Beings of the "Carmela Dutra" maternity ward, Florianópolis / SC under protocol No. 780.303.

\section{Participants}

All students from the 6th to 9th grades (10-16 years of age) who were regularly enrolled in the municipal school system in $2015(\mathrm{n}=7,484)$ were considered eligible for the study. The sample size was calculated according to the effect size, considering Beta $=0.80$ and alfa $=0.05$ for the primary outcomes, assuming the largest required sample $(n=295)$. Five municipal schools were considered eligible because they presented the minimum number of 295 adolescents from the 6th to 9th grades. These five schools were the largest in the city. Two schools were intentionally selected by the Municipal Department of Education for allocation into intervention and control groups, both located in the Northern region of the city; schools were paired by the socioeconomic conditions of the region and comparable physical structure (indoor sports court). Schools in the control and intervention groups had 567 and 411 eligible students, respectively (Figure 1).

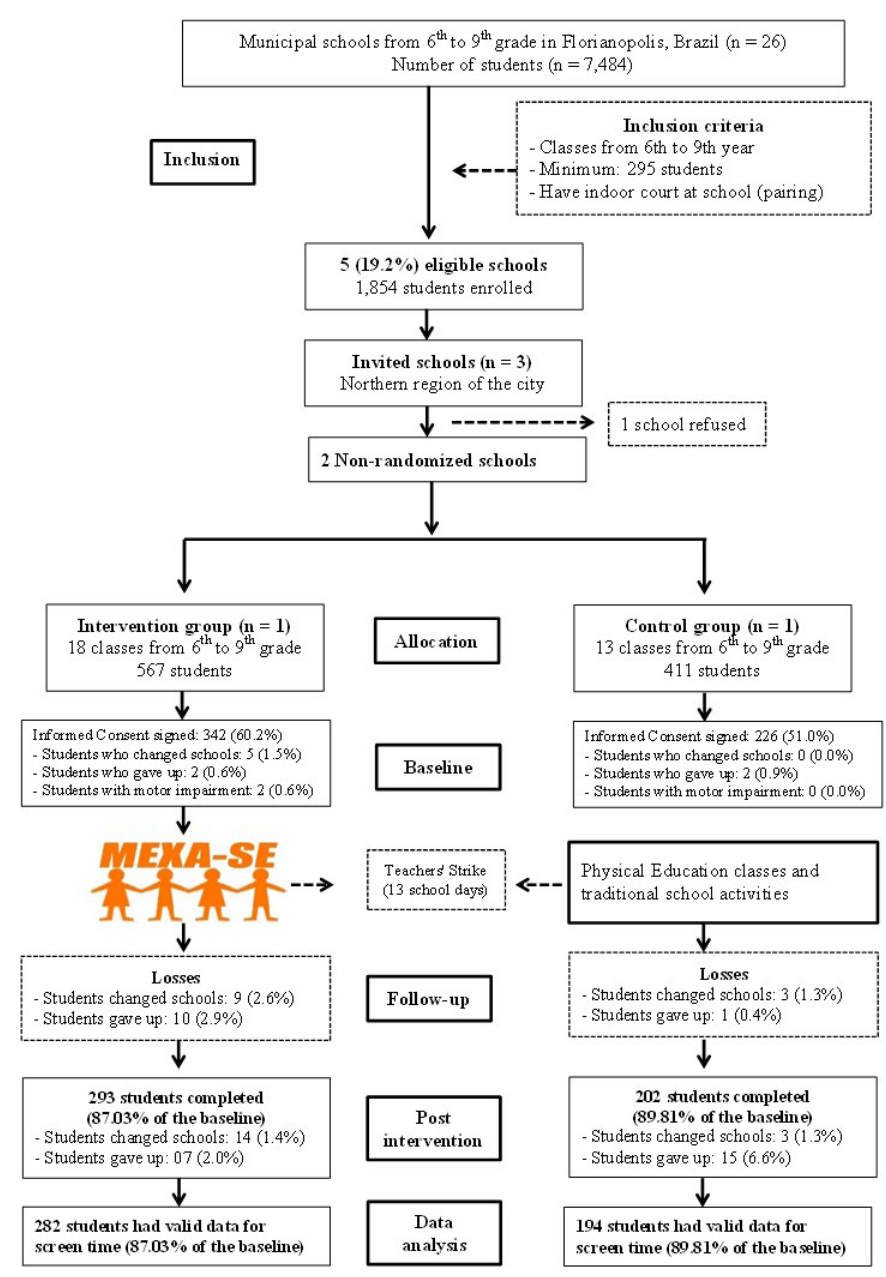

Figure 1. Flow diagram of the MEXA-SE study for the screen time variable.

For the present study, the a posteriori sample calculation was carried out using $\mathrm{G}^{*}$ Power software 3.1.9.2 (Universitat Dusseldorf, Dusseldorf, Germany). Taking into account the final sample with valid data $(\mathrm{IG}=282 ; \mathrm{CG}=194)$, a reduction of $11 \%$ in the screen time of the intervention group and $\alpha=0.05$, there is a power of $75 \%$ for statistical analyses of intragroup comparison (before and after). In addition, the effect size (Cramer's V) was calculated between the baseline groups.

Data collection lasted, on average, 10 days in each phase, which was carried out in March (baseline) and July (postintervention) of 2015 by a previously trained team consisting of 
PE teachers and nutritionists. The application of the questionnaire was developed in a targeted manner and with at least two evaluators in the classroom. While one evaluator performed the reading of the instrument, the others were available to resolve any doubts. The time required for the application of the complete study questionnaire ranged from one to two lesson hours (45 and 90 minutes, respectively).

\section{Intervention}

Screen time was the secondary outcome of the intervention; however, many strategies adopted aimed at reducing it. The "MEXA-SE" multicomponent intervention was developed through four main components, including PE classes, active recess, educational sessions and educational materials. The intervention duration was planned for 14 weeks; however, it was effectively carried out for 11 school weeks due to the teachers' strike in the municipal school network and the delay of the municipal department of education to allocate schools in $\mathrm{IG}$ and $\mathrm{CG}$.

\section{Intervention strategies}

The "PE class" component proposed changes in the structure of classes that lasted 45 minutes and should allow 20 minutes for aerobic activities, 10 minutes for strength and muscular endurance activities and 10 minutes for flexibility activities. PE teachers were responsible for planning and giving classes in the format suggested by the intervention. Active recess consisted of the availability of sports materials (balls and ropes) for use in the school spaces during the recess period.

Four educational sessions on body image were developed, lasting one lesson hour each (45 minutes), dealing with satisfaction about self-image, beauty standards imposed by the media, and the construction of positive self-esteem. To this end, sessions had beauty standards and individual qualities as themes, addressed by means of photographic session, discussions and elaboration of posters. Nutrition sessions, six in total, lasting approximately $1 \mathrm{~h} 40 \mathrm{~min}$ (each), addressed topics related to healthy eating as a way of promoting health and disease prevention. Examples includechoosing fresh foods, vegetable consumption and cooking skills, which were addressed through lectures, group discussions and workshops.

Two sessions, focused on health, lifestyle, physical activity and sedentary behavior were developed: one in the third and the other in the fifth week of the intervention, each lasting one lesson hour (45 minutes) each and applied by PE teachers. These sessions aimed to identify the behaviors present in the daily life of adolescents, seeking to clarify concepts and discuss the relationship between adopted behaviors, health and healthy lifestyle. For that, videos, games, and group discussions were used.

The distribution of illustrative materials characterizes the fourth component of the intervention, which was carried out during the school period and aimed to reinforce the content previously presented in educational sessions. Two folders with different contents were distributed to adolescents and two were sent to parents and/or guardians, containing information about: 1) the importance of physical activity and health benefits; 2) incentive to parents' practice of physical activity and its importance; and 3) the need to control time in the use of television, computer and video games. Posters containing information on how to reduce screen time and increase physical activity were exposed in the school environment during the intervention with the intention of reinforcing messages to adolescents, teachers and parents, in view of their potential as informational and social support vehicles. The strategies developed in the present intervention were based on elements of theories for behavioral change. Constructs of the Cognitive Social Theory ${ }^{21}$ and HealthPromoting Schools ${ }^{22}$ guided the planning of educational sessions, folders and posters (Figure 2).
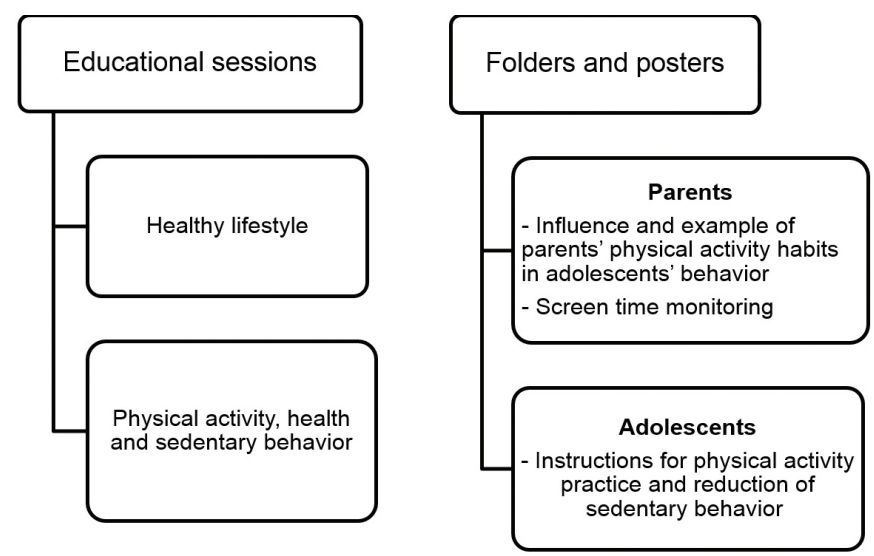

Figure 2. Organizational chart of actions applied in the "MEXASE" intervention specifically focused on screen time.

\section{Group control}

The CG remained with usual school activities, with three weekly PE classes (45 minutes each).

\section{Study variables}

All variables in this study were self-reported by students. Screentime was estimated by calculating the variables of time in front of television (TV), computer and/or video game (CPT/VG) on weekdays and weekends. From the question "In general, how many hours per day do you watch TV/use the Computer/Videogame?" The answer alternatives were "I do not watch TV/CPT/VG", "less than 1 hour per day", "1 hour per day", "2 hours per day", "3 hours per day", "4 hours per day" or "5 hours or more per day" on weekdays and weekends based on the Youth Risk study questionnaire 
Behavior Survey (YRBS) ${ }^{23}$ and validated for the Brazilian adolescent population ${ }^{24}$. Variable screen time was dichotomized into $<2$ hours or $\geq 2$ hours. The cutoff point for excess screen time was two hours or more exposed to any of the behaviors investigated ${ }^{3,25}$. Screen behaviors were treated separately and there was no sum of the total time spent in these activities.

Considering that the literature indicates that socioeconomic level and age are factors influencing screen-time ${ }^{26}$, these characteristics were considered in the study as adjustment variables. Socioeconomic information was obtained through a questionnaire, estimated by purchasing power and classified according to the criteria of the Brazilian Association of Research Companies ${ }^{27}$. Economic levels with low frequency were grouped, resulting in "A and $B$ ", "C" and " $D$ and $E "$, considered in descending order of purchasing power, in which categories A and B present the greatest power and categories $\mathrm{D}$ and $\mathrm{E}$ the lowest purchasing power.

\section{Statistical analysis}

Descriptive statistics were used to verify the absolute and relative frequencies for each variable. At baseline, the chi-square test was used to verify the similarity between intervention and control groups for sex, economic level, age group and screen time (TV, CPT/VG in weekdays and weekends). Intragroup differences in the proportion of adolescents in excess screen time from baseline to post-intervention were tested using the McNemar test. Binary logistic regression analysis was used to develop crude models and were adjusted by screen time at baseline, age and socioeconomic level. The odds ratio and the confidence interval (CI95\%) of change in the proportions of excess screen time (dependent variable) were estimated considering the baseline for after intervention and group (intervention $=1$ and control $=0$ ) as independent variables. Analyses were stratified by sex. Only complete data for screen time were analyzed, no intention-to-treat analysis was applied, considering the small sample loss after intervention. The "Statistical Package for the Social Sciences" software (SPSS IBM Inc., Chicago, USA) version 22 was used in all analyses considering $\mathrm{p}<0.05$.

\section{Results}

The sample consisted of 476 adolescents ( 282 in the intervention group [IG]). The response rate at the end of the intervention was $87.03 \%(\mathrm{n}=282)$ in IG and $89.81 \%(\mathrm{n}=194)$ in the control group (CG) in relation to baseline (data not presented in tables/figures).

Table 1 shows that IG and CG were similar at baseline in relation to demographic, economic, and screen time variables and the effect size between the groups was weak at baseline. The frequency of excess TV, CPT/VG, during the week and weekend ranged from 54.4 to $75.3 \%$ in both groups (Table 1 ).

Table 1. Comparison between groups (intervention and control) at baseline. "MEXA-SE” Study, Florianópolis, SC / Brazil, 2015

\begin{tabular}{|c|c|c|c|c|c|}
\hline & $\begin{array}{c}\text { Intervention } \\
\% \text { (n) }\end{array}$ & $\begin{array}{c}\text { Control } \\
\% \text { (n) }\end{array}$ & $\mathbf{X}^{2}$ & $\mathbf{P}$ & Cramer's V \\
\hline \multicolumn{6}{|l|}{ Sex } \\
\hline Male & 45.7 (148) & $48.6(105)$ & \multirow[t]{3}{*}{0.447} & \multirow[t]{3}{*}{0.504} & \multirow[t]{3}{*}{0.03} \\
\hline Female & $54.3(176)$ & $51.4(111)$ & & & \\
\hline $\mathrm{A}+\mathrm{B}$ & 60.8 (197) & $58.8(127)$ & & & \\
\hline $\mathrm{C}$ & 36.4 (118) & $39.4(85)$ & \multirow[t]{2}{*}{0.845} & \multirow[t]{2}{*}{0.655} & \multirow[t]{2}{*}{0.04} \\
\hline $\mathrm{D}+\mathrm{E}$ & $2.8(9)$ & $1.9(4)$ & & & \\
\hline \multicolumn{6}{|l|}{ Age Group } \\
\hline $10-12$ & $49.2(159)$ & $51.9(112)$ & \multirow{2}{*}{0.357} & \multirow{2}{*}{0.550} & \multirow{2}{*}{0.03} \\
\hline $13-16$ & $58.8(164)$ & 48.1(104) & & & \\
\hline \multicolumn{6}{|l|}{ BMI } \\
\hline Normal weight & 69.3 (217) & $65.1(136)$ & \multirow{2}{*}{1.037} & \multirow{2}{*}{0.308} & \multirow{2}{*}{0.05} \\
\hline Overweight & $30.7(96)$ & $34.9(73)$ & & & \\
\hline \multicolumn{6}{|l|}{ Screen time } \\
\hline \multicolumn{6}{|l|}{ TV/Week } \\
\hline$<2 \mathrm{hs}$ & $24.7(80)$ & $30.6(66)$ & 2.259 & 0.133 & 0.07 \\
\hline$\geq 2 \mathrm{hs}$ & $75.3(244)$ & $69.4(150)$ & & & \\
\hline \multicolumn{6}{|l|}{ TV/Weekend } \\
\hline$<2 \mathrm{hs}$ & $37.8(122)$ & $38.9(84)$ & 0.069 & 0.794 & 0.01 \\
\hline$\geq 2 \mathrm{hs}$ & $62.2(201)$ & $61.1(132)$ & & & \\
\hline
\end{tabular}




\begin{tabular}{|c|c|c|c|c|c|}
\hline CPT-VG / Week & & PT-VG / Wee & \multirow{3}{*}{0.078} & \multirow{3}{*}{0.780} & \multirow{3}{*}{0.01} \\
\hline$<2 \mathrm{hs}$ & $43.1(140)$ & $41.9(90)$ & & & \\
\hline$\geq 2 \mathrm{hs}$ & $56.9(185)$ & $58.1(125)$ & & & \\
\hline \multicolumn{6}{|c|}{ CPT - VG/ Weekend } \\
\hline$<2 \mathrm{hs}$ & $44.2(141)$ & $45.6(98)$ & 0.099 & 0.753 & 0.01 \\
\hline$\geq 2 \mathrm{hs}$ & $55.8(178)$ & $54.4(117)$ & & & \\
\hline
\end{tabular}

TV: television; CPT and / or VG: computer and / or video game; BMI: Body Mass Index (WHO)

In the pre and post intervention intragroup, it was found that in males, there was a reduction of CPT/VG time on weekdays by about $14.0 \%$. This association was considered weak in terms of effect size. In females, there was a decline of $8.9 \%$ in TV time on weekdays. There was no change from baseline to post-intervention in the CG. For the other electronics, there was no significant reduction in screen time in both IG and CG (Table 2).
Logistic regression was used to test the reduction of the proportion of adolescents exposed to excess screen time in IG compared to CG. Analyses were adjusted for economic level, age and screen time at baseline. There were no significant results in the odds ratio of reducing the proportion of adolescents' excess screen time, when comparing the IG with the GC (Table 3).

Table 2. Relative and absolute frequency of excess screen time ( $\geq 2$ hours) for television, computer and / or videogame in the pre and post-intervention control and intervention groups, separated by sex. "MEXA-SE” Study, Florianópolis, SC / Brazil, 2015.

\begin{tabular}{|c|c|c|c|c|c|c|c|c|c|c|}
\hline & \multicolumn{5}{|c|}{ Intervention group } & \multicolumn{5}{|c|}{ Control Group } \\
\hline & Pre & Post & $\Delta$ & $\mathbf{P}^{*}$ & Cramer's V & Pre & Post & $\Delta$ & $\mathbf{P}^{*}$ & Cramer's V \\
\hline \multicolumn{11}{|l|}{ Boys } \\
\hline & $\%(\mathrm{n})$ & $\%(\mathrm{n})$ & & & & $\%(n)$ & $\%(\mathrm{n})$ & & & \\
\hline TV/Week & $70.5(86)$ & $68.0(83)$ & -2.5 & 0.72 & 0.02 & $65.1(56)$ & $72.1(62)$ & 7.0 & 0.31 & 0.16 \\
\hline TV/ Weekend & $65.8(79)$ & $60.8(73)$ & -5.0 & 0.35 & 0.04 & $55.3(47)$ & $62.4(53)$ & 7.1 & 0.33 & 0.06 \\
\hline $\begin{array}{l}\text { CPT and/or } \\
\text { VG / Week }\end{array}$ & $71.7(86)$ & $57.5(69)$ & -14.2 & 0.01 & 0.12 & $64.0(55)$ & $61.6(53)$ & 2.4 & 0.82 & 0.02 \\
\hline $\begin{array}{l}\text { CPT and/or } \\
\text { VG / Weekend } \\
\text { Girls }\end{array}$ & $68.9(82)$ & $64.7(77)$ & -4.2 & 0.44 & 0.03 & $61.4(51)$ & $65.1(54)$ & 3.7 & 0.52 & 0.03 \\
\hline TV/Week & 81.5 (119) & $72.6(106)$ & -8.9 & 0.02 & 0.08 & $71.8(74)$ & $73.8(76)$ & 2.0 & 0.82 & 0.02 \\
\hline TV/ Weekend & $62.3(91)$ & $64.4(94)$ & 2.1 & 0.76 & 0.04 & $65.0(67)$ & $67.0(69)$ & 2.0 & 0.84 & 0.02 \\
\hline $\begin{array}{l}\text { CPT and/or } \\
\text { VG / Week }\end{array}$ & $44.8(65)$ & $44.8(65)$ & 0.0 & 1.00 & 0.00 & $47.1(48)$ & $36.3(37)$ & -10.8 & 0.09 & 0.10 \\
\hline $\begin{array}{l}\text { CPT and/or } \\
\text { VG / Weekend }\end{array}$ & $43.1(62)$ & $42.4(61)$ & -0.7 & 1.00 & 0.01 & $44.7(46)$ & $34.0(35)$ & -10.7 & 0.07 & 0.10 \\
\hline
\end{tabular}

TV: television, CPT and / or VG: computer and / or video game. *Comparison of proportions by the McNemar test

Table 3. Odds ratio for excess screen time ( $\geq 2$ hours) of adolescents in the intervention group, using the control group in the "MEXA-SE" study as reference, Florianópolis, SC / Brazil, 2015.

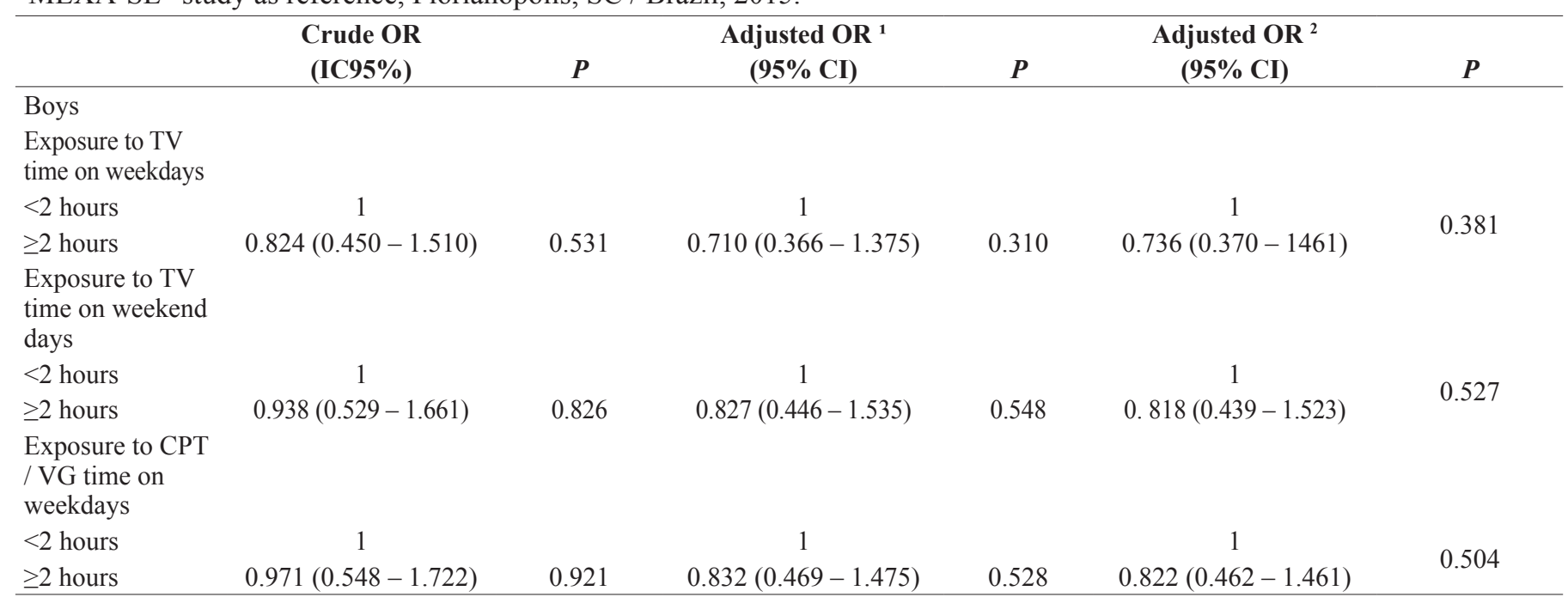




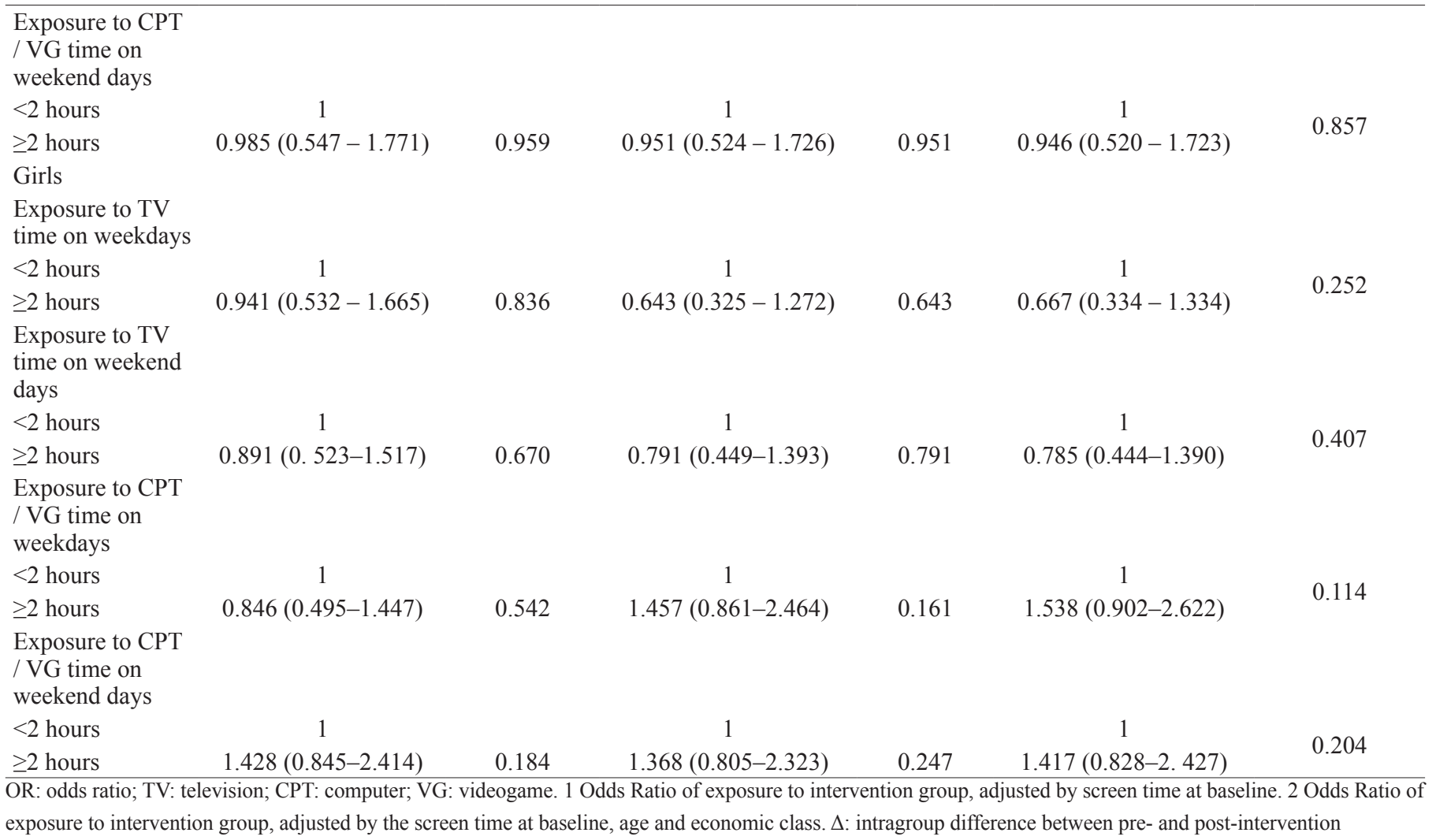

\section{Discussion}

The main result of the present study was to reduce computer and / or videogame time in male adolescents and television time in female adolescents from the intervention group on weekdays based on a multicomponent intervention, applied in school reality, supported by the behavioral change theory (and health at school) and applied to adolescents of both sexes. Although the intervention effectiveness was not found for the comparison between groups, the intervention characteristics allow the implementation in several contexts, especially in places with limited resources, due to the low cost and development supported by the actions of the school staff, itself.

Reducing screen time improves health indicators ${ }^{1-3}$. A systematic review 1 of data from 1.5 million participants (children and adolescents) from around the world, indicate that a higher duration of TV viewing and/or screen time was associated with unfavorable health (e.g., obesity, cardiovascular disease risk factors, unfavorable measures of behavioral conduct, lower health-related physical fitness, lower self-esteem) and reductions in screen time improves these indicators ${ }^{1}$. The MEXA-SE protocol is easy to apply and seems to be more suitable for reducing screen time in adolescents than traditional Physical Education classes. This was because the MEXA-SE protocol was built on theories of behavioral change ${ }^{21,22}$. Such theories are not employed in the traditional Physical Education classes and, it is for this reason that traditional Physical Education classes represent very little in the change of behavior of the adolescents.

The results found in this study corroborated previous multicomponent school-based interventions that used educational strategies effective in reducing screen time in adolescents ${ }^{13,17}$. The reduction in the computer and / or video game time observed in male adolescents and that of television time in female from the IG corroborates the hypothesis that individuals most exposed to risk behavior tend to assimilate educational messages more positively. The literature indicates sedentary behavior based on the use of screens (e.g., computer and videogame), more representative among boys ${ }^{26}$, and television, among girls ${ }^{6}$. Considering that males are more exposed to computer and videogame use, a previous study ${ }^{14}$ has shown a reduction in the use of these electronics. The present intervention resembles the study by Cui et al. ${ }^{14}$, regarding the methodological strategies adopted to reduce screen time, particularly the use of educational sessions, active games and group discussions, and the use of the Sociocognitive Theory. However, intervention ${ }^{14}$ was carried out by the peers themselves - from older students to younger students - instead of researchers, which positively influenced the reduction in the computer and video game time also in female adolescents, as well as in other studies ${ }^{13,17}$ and different from that observed in this intervention.

Regarding the reduction in the use of TV among females, the findings of the present intervention corroborate data from previous studies ${ }^{15,16,28}$, since the present study verified a mean reduction of $8.9 \%$ of individuals exposed to long periods in front of the TV screen. The methodological options of this study were similar to classic studies that promoted reduction in TV time in adolescents ${ }^{15,16}$, especially in the use of educational sessions developed in classrooms and educational material sent 
to parents of adolescents to help reducing screen time.

In both behaviors (TV and computer) and sexes (male and female), exposure is considerably higher on weekends when compared to weekdays. The extensive demand for school and homework on weekdays increases sedentary behavior not related to the use of screens, such as reading and writing, and reduces the time spent on leisure screen time (television, computer and video game) in the adolescent's daily life ${ }^{26}$. However, the increase in discretionary time on weekends can influence the behavior choice and, consequently, increase the exposure to television and computer screens ${ }^{29}$. This behavioral trait may explain the difficulty in finding changes in screen time on weekends and positive results in reducing exposure on weekdays found in the present study.

Behavioral changes are difficult to implement and to present significant results ${ }^{30}$. Although no significant screen time changes were observed in IG compared to $\mathrm{CG}$, the observed effect on intragroup changes from baseline to post-intervention demonstrates an important result that contributes to the current list of school interventions applied in middle-income countries. Educational strategies such as those used in low-cost "MEXASE", which can be easily reproduced by PE teachers and other school teachers, seem to be able to reduce screen time.

The duration of the present intervention was relatively short and may be considered a limitation, given that behavioral changes occur in a temporal way, and include several stages that often range from not intending to modify it to the self-efficacy of keeping it. The intervention was planned for 14 weeks; however, it was developed in 11 weeks due to the teachers' strike in the municipal teaching network of the city under investigation. This political-labor scenario is representative of the Brazilian reality and of other countries worldwide, and is of extreme importance to be considered in intervention studies on screen time. Another limitation is that the sample power was less than $80 \%$ for the statistical analysis which represented weak effects sizes for the results. The lack of follow-up of participants to verify behavioral changes and the use of a questionnaire (without being combined with an objective measure) to verify the screen time -- that has memory bias - are also limitations. The absence of randomization in the allocation of schools was another study limitation, which does not guarantee that the causality observed occurred at random.

The possibilities of analysis of sedentary behavior vary according to the way information is collected, which can be collected objectively or subjectively. Objective analysis is done by means of motion sensors such as accelerometers; subjective measurement is obtained through questionnaires. In discretionary activities, such as time in front of screens at leisure, the literature points out the use of questionnaires as the main research source used ${ }^{3}$ as well as the treatment of time-of-screen variables in a categorical way, using the cutoff point of two hours to characterize excess screen time ${ }^{25}$. The measurement of screen time by questionnaires is valid and used way in other studies ${ }^{10,11}$. However, this measure fails to capture the process of behavioral change. The fact that the intervention presents strategies of Health Education can modify the attitude and knowledge of the adolescents in relation to screen-time, but such factors are not captured by the questionnaire.

Our study has opted analyses OR estimates rather than risk ratios (RR). This decision may generate doubts for some experts, but it was made based on recommendations in the literature ${ }^{31,32}$. We opted for OR for the following reasons: 1) it is more symmetrical than RR; 2) we did not aim to compare the magnitudes of association between the different outcomes for screen-time; 3) as we opted for the OR, our covariates should be similar between the baseline groups, and this occurred. For more details on this debate, the literature brings interesting information that can be consulted ${ }^{31,32}$.

The strengths of the study include the use of elements of Behavior Change Theories to support the planning of the intervention, the multicomponent character represented by the different approaches of the outcome, focusing on the subject, parents and the school environment, as well as the development of an intervention with adolescents living in a middle-income country.

From a practical application perspective of these findings, the "MEXA-SE" intervention provides tools that can reduce screen time such as sessions and low-cost educational materials delivered by the school's managers and faculty, representing a way to intervene in a large group of adolescents from the current school structure. Given the high number of adolescents exposed to excess screen time, which may represent a public health problem, effective strategies may contribute to a change in this scenario.

\section{Conclusion}

The "MEXA-SE" multi-component intervention based on health education strategies was not effective in reducing screen time in the comparison between intervention and control groups. Further studies focusing on different strategies, combined objective and subjective sedentary behavior measures and larger samples are needed to identify possible differences between intervention and control groups. The "MEXA-SE" intervention found a promising result which was the reduction of computer and television time on weekdays in male and female adolescents, respectively, in the intervention groups. This finding may be indicative that the intervention process may have resulted in changes in attitudes and knowledge of adolescents.

\section{Acknowledgments}

To the National Council of Scientific and Technological Development (CNPq) for the macroproject financing. To all students, teachers, managers and parents who accepted to be part of the present study. The entire "MEXA-SE" work team holding hands for health". 


\section{References}

1. Carson V, Hunter S, Kuzik N, Gray C, Poitras V, Chaput J, et al. Systematic review of sedentary behaviour and health indicators in school-aged children and youth: an update. Appl Physiol Nutr Metab. 2016;41(6 (Suppl. 3):S240-S265

2. Grøntved A. Television Viewing and Risk of Type 2 Diabetes, Cardiovascular Disease, and All-Cause Mortality. JAMA. 2011;305(23):2448.

3. Tremblay M, LeBlanc A, Kho M, Saunders T, Larouche R, Colley R, et al. Systematic review of sedentary behaviour and health indicators in school-aged children and youth. Int J Behav Nutr Phys Act. 2011;8(1):98.

4. Saunders T, Chaput J, Tremblay M. Sedentary Behaviour as an Emerging Risk Factor for Cardiometabolic Diseases in Children and Youth. Can J Diabetes. 2014;38(1):53-61.

5. World Health Organization. Inequalities in young people's health: HBSC international report from the 2005/2006 survey. Copenhagen; 2008.

6. Instituto Brasileiro de Geografia e Estatística. Pesquisa Nacional de Saúde do Escolar - PeNSE 2012. Rio de Janeiro; 2013.

7. Dias P, Domingos I, Ferreira M, Muraro A, Sichieri R, Gonçalves-Silva R. Prevalência e fatores associados aos comportamentos sedentários em adolescentes. Rev Saude Publica. 2014;48(2):266-274.

8. Andrade S, Lachat C, Ochoa-Aviles A, Verstraeten R, Huybregts L, Roberfroid D, et al. A school-based intervention improves physical fitness in Ecuadorian adolescents: a cluster-randomized controlled trial. Int J Behav Nutr Phys Act. 2014;11:153.

9. Bergh I, van Stralen M, Bjelland M, Grydeland M, Lien $\mathrm{N}$, Klepp K, et al. Post-intervention effects on screen behaviours and mediating effect of parental regulation: the HEalth In Adolescents study - a multi-component school-based randomized controlled trial. BMC Public Health. 2014;14:200.

10. Harrison M, Burns C, McGuinness M, Heslin J, Murphy $\mathrm{N}$. Influence of a health education intervention on physical activity and screen time in primary school children: 'Switch Off-Get Active'. J Sci Med Sport. 2006;9(5):388-94.

11. Salmon J, Ball K, Hume C, Booth M, Crawford D. Outcomes of a group-randomized trial to prevent excess weight gain, reduce screen behaviours and promote physical activity in 10-year-old children: Switch-Play. Int J Obes. 2008;32(4):601-12.

12. Singh A, Chin A Paw M, Brug J, van Mechelen W. Dutch obesity intervention in teenagers: effectiveness of a schoolbased program on body composition and behavior. Arch Pediatr Adolesc Med. 2009;163(4):309-17.

13. Dewar D, Morgan P, Plotnikoff R, Okely A, Batterham M, Lubans D. Exploring changes in physical activity, sedentary behaviors and hypothesized mediators in the NEAT girls group randomized controlled trial. J Sci Med Sport. 2014;17(1):39-46. .
14. Cui Z, Shah S, Yan L, Pan Y, Gao A, Shi X, et al. Effect of a school-based peer education intervention on physical activity and sedentary behaviour in Chinese adolescents: a pilot study. BMJ Open. 2012;2(3):e000721.

15. Gortmaker S, Peterson K, Wiecha J, Sobol A, Dixit S, Fox M, et al. Reducing Obesity via a School-Based Interdisciplinary Intervention Among Youth. Arch Pediatr Adolesc Med. 1999;153(4):409-18.

16. Young D, Phillips J, Yu T, Haythornthwaite J. Effects of a Life Skills Intervention for Increasing Physical Activity in Adolescent Girls. Arch Pediatr Adolesc Med. 2006;160(12):1255-61.

17. Leme A, Lubans D, Guerra P, Dewar D, Toassa E, Philippi S. Preventing obesity among Brazilian adolescent girls: Six-month outcomes of the Healthy Habits, Healthy Girls-Brazil school-based randomized controlled trial. Prev Med. 2016;86:77-83.

18. da Silva L, Fisberg M, de Souza Pires M, Nassar S, Sottovia C. The effectiveness of a physical activity and nutrition education program in the prevention of overweight in schoolchildren in Criciúma, Brazil. Eur J Clin Nutr. 2013;67(11):1200-4.

19. Hardman C, Barros M, Lopes A, Lima R, Bezerra J, Nahas M. Efetividade de uma intervenção de base escolar sobre o tempo de tela em estudantes do ensino médio. Rev Bras Cineantropom Desempenho Hum. 2014;16(Suppl 1):25-35.

20. Friedrich R, Polet J, Schuch I, Wagner M. Effect of intervention programs in schools to reduce screen time: a meta $\square$ analysis. J Pediatr (Rio J). 2014;90(3):232-41.

21. Bandura A. Social cognitive theory. In Annais of child development. Six theories of child development. Greenwich; 1989. p. 1-60.

22. Ministério da Saúde. Escolas promotoras de saúde: experiências do Brasil. Brasília: Organização PanAmericana de Saúde; 2007.

23. YRBS Questionnaire Content 1991-2017 [Internet]. EUA: Centers for Disease Control and Prevention; 2016 [cited 19 November 2017]. Available from: https://www.cdc. gov/healthyyouth/data/yrbs/pdf/2017/yrbs_questionnaire content_1991-2017.pdf

24. Barbosa Filho V, Lopes A, Lima A, de Souza E, Gubert F, Silva K, et al. Rationale and methods of a clusterrandomized controlled trial to promote active and healthy lifestyles among Brazilian students: the "Fortaleça sua Saúde" program. BMC Public Health. 2015;15(1).

25. American Academy of Pediatric. Children, adolescents, and television. EUA: Committee on public education; 2001 p. 423-6.

26. Olds T, Maher C, Ridley K, Kittel D. Descriptive epidemiology of screen and non-screen sedentary time in adolescents: a cross sectional study. Int J Behav Nutr Phys Act. 2010;7(1):92.

27. Associação Brasileira de Empresas de Pesquisa (ABEP). Critério de Classificação Econômica. Brasil; 2012. 
28. Chavarro J, Peterson K, Sobol A, Wiecha J, Gortmaker S. Effects of a School-based Obesity-prevention Intervention on Menarche (United States). Cancer Causes Control 2005;16(10):1245-52.

29. Biddle S, Gorely T, Marshall S. Is television viewing a suitable marker of sedentary behavior in young people? Ann Behav Med. 2009;38(2):147-53.

\section{Corresponding author}

Diego Augusto Santos Silva

Universidade Federal de Santa Catarina, Centro de Desportos,

Departamento de Educação Física, Campus Universitário, Trindade,
Caixa Postal 476, Florianópolis, Santa Catarina, Brasil.

Email: diegoaugustoss@yahoo.com.br

Manuscript received on March 23, 2018

Manuscript accepted on June 18, 2018

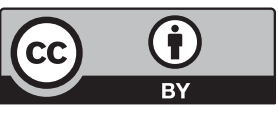

Motriz. The Journal of Physical Education. UNESP. Rio Claro, SP, Brazil - eISSN: 1980-6574 - under a license Creative Commons - Version 3.0 